\title{
The Rougher The Better. EINE GESCHICHTE DES > DRECKIGEN SOUNDS<, SEINER ÄSTHETISCHEN MOTIVE UND SOZIALEN FUNKTIONEN
}

\author{
Ralf von Appen
}

Lässt man die Geschichte der Pop- und Rockmusik mit dem Fokus auf besondere Momente der Soundentwicklung Revue passieren, so fällt die Aufmerksamkeit schnell auf prominente Produzenten wie Phil Spector, Brian Wilson oder George Martin, die Mitte der 1960er Jahre für aufwändig hergestellte, noch heute als Meilensteine geltende Aufnahmen verantwortlich waren. Fortschritte in der Studiotechnik und das Bestreben, den Gesamtklang der Aufnahmen vom Akzidens zu einem wesentlichen Bereich der kreativen Gestaltung zu machen, bedingten sich in diesen Jahren gegenseitig. Die Verfügbarkeit der Mehrspurtechnik und verschiedenster Geräte zur Klangoptimierung oder -verfremdung im Studio sowie die wachsende Verbreitung von HiFi-Stereo-Anlagen bei den Hörern setzten neue Standards für einen sguten Sound. Man erwartete und produzierte zunehmend brillante, durchsichtige Aufnahmen mit differenzierten künstlichen Klangräumen, die erstmals besser und realistischer klangen als Livekonzerte (bspw. der Beatles).

Doch sobald solche aufwändigen, Perfektion anstrebenden Produktionen zur ästhetischen Norm wurden, begannen Musiker auch, die neuen Standards bewusst zu unterlaufen oder die Technik entgegen der eigentlichen Intention zu nutzen, um besonders raue, unrealistische, weithin als shässlich $<$ angesehene Klangbilder zu erzielen. Sie verzichteten auf Wohlklang und damit zugleich auf kommerziellen Erfolg, waren aber gerade wegen ihrer Soundgestaltung nicht weniger einflussreich für die Entwicklung der Rockmusik. Im Folgenden soll eine solche Traditionslinie unangepasster, nicht an den Idealen von Schönheit und Klarheit orientierter Soundproduktion bis in die Gegenwart nachgezeichnet werden, wobei das Interesse neben den geschichtlichen Bezügen auch den ästhetischen Motiven und den sozialen Funktionen solcher Musik gilt. 
Während Rock'n'Roll-Musiker der 1950er Jahre noch kaum andere Chancen hatten, als so zu klingen, wie es die Beschränkungen der Technik oder das geringe Budget gerade erlaubten, fällt bei den sogenannten Garagen-Bands (benannt nach dem Hauptschauplatz ihres Wirkens) die Entscheidung schwer, wann sich ihr Sound durch mangelnde Mittel und/oder Fähigkeiten und wann durch bewusste Absicht begründet. Beeindruckt von der British Invasion hatten sich um 1964 weiße Vorstadt-Teenager vor allem in Kalifornien und Texas zu Gruppen wie The Kingsmen, The Barbarians, The Seeds, The Count Five oder The Standells zusammengetan, um die Energie und die Wildheit der Beatles, Rolling Stones, Kinks, Animals oder Who nachzuahmen. Mangelnde musikalische Fähigkeiten wurden dabei durch Enthusiasmus, erhöhtes Tempo und Lautstärke aufgefangen (vgl. Unterberger o.J.[a]; Bangs 1992). Vor allem Aufnahmen der Sonics - bspw. »The Witch« (1964) oder »Strychnine « (1965) - klingen wie zufällig mitgeschnittene ProberaumSessions mit schmerzend scheppernder Snare-Drum, wilden Schreien, einem sehr dünnen Bassklang und beeindruckend verzerrter Gitarre, deren rauer Sound kaum vom ebenfalls im Hintergrund spielenden Saxofon zu unterscheiden ist. Ihre kraftvollen Versionen von »Money (That's What I Want)《 oder »Louie Louie« (beide 1965) zeigen allerdings gerade im Vergleich mit den Aufnahmen dieser Stücke von den Beatles, den Kinks oder den Kingsmen, was technisch möglich gewesen wäre und wie wenig es den Sonics offenbar um ssaubere`, den Ansprüchen von Radiosendern genügende Aufnahmen ging. Gleiches ließe sich am schrill-überdrehten »Hey Joe « von den Leaves im Vergleich zu den Versionen der Shadows of Knight oder der Jimi Hendrix Experience (alle 1966) zeigen.

Weder aus jugendlichem Überschwung noch aus spieltechnischem Unvermögen entstand dagegen der Sound der frühen Velvet Underground, deren ersten beiden LPs als Beginn (und möglicherweise auch radikaler Höhepunkt) der Tradition bewusst kruder Sounds angesehen werden können. Zwar waren auch die Etats für die Produktion der damals überraschend erfolglosen LPs The Velvet Underground \& Nico (Platz 171 in den Billboard Charts) und White Light / White Heat (Platz 199) nicht mit den Möglichkeiten etwa der Beatles zu vergleichen, die sich als Haupteinnahmequelle der EMI um Studiokosten nicht zu sorgen brauchten: Während das Sgt. PepperAlbum zwischen Dezember 1966 und April 1967 in ca. 700 Stunden Studioarbeit zusammengebastelt werden konnte, schnitten Velvet Underground ihr Debüt zunächst an ein oder zwei Tagen im April 1966 unter der Aufsicht Andy Warhols für 1500 Dollar im heruntergekommenen New Yorker Scepter 
Records Studio mit. ${ }^{1}$ Doch für die musikalischen Ziele der Band reichten die spartanischen Möglichkeiten aus. Ihr ästhetisches Konzept speiste sich aus verschiedenen künstlerischen Avantgarden, die sie auf die Rockmusik übertragen wollten. Wesentlich waren dafür die Ideen ihres Mentors Andy Warhol und die Arbeiten des Minimalisten La Monte Young, zu dessen Improvisationsgruppe John Cale von 1963 bis 1965 gehört hatte. ${ }^{2}$

Das siebenminütige »Heroin« vom Debüt z.B. folgt der Leitidee des Minimalismus nicht nur in der Produktionstechnik, sondern in allen musikalischen Parametern. Das Arrangement beschränkt sich neben dem monotonen Gesang Lou Reeds auf eine einzige Trommel mit dem Klang einer leeren Waschmittelpackung, eine (manchmal verzerrte) E-Gitarre, die ausschließlich zwischen Tonika und Subdominante wechselt, die Viola John Cales, der im Wesentlichen einen einzigen sirenenartigen Ton spielt, sowie kreischendes Feedback. Andere Songs klingen wie Live-Mitschnitte ohne jegliche Bemühungen, den Klang nach der Aufnahme durch Effektgeräte oder differenziertes Abmischen den üblichen Standards der Radiosender anzupassen. Mit einem Einsatz im Radio konnten die Musiker freilich ohnehin nicht rechnen, standen doch Texte und Musik im größtmöglichen Gegensatz zur bürgerlichen Kultur und zu den subkulturell vorherrschenden Hippie-Idealen von harmonischer Liebe, Frieden, Natürlichkeit, durch Drogen befreites und erweitertes Bewusstsein oder Hoffnungen auf eine neue, bessere Gesellschaft. Stattdessen sang Lou Reed lakonisch realitätsnahe Texte über Sadomasochismus, Heroinabhängigkeit und die dunklen Seiten des Lebens in New York. So wird es Ziel der Soundgestaltung gewesen zu sein, der harten, radikalen Wirklichkeit der Lyrics ein korrespondierendes Klanggewand zu schaffen.

Noch konsequenter als auf dem Debüt, wo zumindest die Balladen in melancholisch gefärbtem Wohlklang erschienen, bemühten sich die Musiker

1 Einige Songs (»Heroin«, »Venus In Furs«, »I'm Waiting For The Man« und »Sunday Morning «) spielte man dann kurz darauf unter dem Produzenten Tom Wilson (Bob Dylan, Simon \& Garfunkel) noch einmal neu ein. Die Gelegenheit zur Veröffentlichung ergab sich erst ein Jahr später, im März 1967.

2 Vgl. John Cale: »Der Sound von Velvet Underground kam von der Arbeit mit La Monte Young im Theater of Eternal Music. Wir fanden heraus, welch großartigen Orchesterlärm wir mit einem Bogen aus einer Gitarre herausholen konnten. Das übertrugen wir auf Viola und Geige. Ich feilte den Steg einer Viola ab und spielte auf drei Seiten ... Das verursachte großartigen Lärm. Es klang so, als würde ein Flugzeug durchs Zimmer fliegen « (zit. in Palmer 1997: 193). Cale hatte zudem mit Cage und Copland zusammengearbeitet. Des Weiteren finden sich auch Free Jazz-Einflüsse, etwa in Reeds feedbackgetränkten Gitarrensoli bei »I Heard Her Call My Name«, in dessen schnellen atonalen Läufen über den gesamten Gitarrenhals David Fricke (1992: 355) sich an Cecil Taylor und Ornette Coleman erinnert fühlt. 
auf dem im September 1967 aufgenommenen Album White Light / White Heat (veröffentlicht im Januar 1968) um ein brachiales, rohes Erscheinungsbild: "The first one had some gentility, some beauty. The second one was consciously anti-beauty«, bringt John Cale das Ergebnis auf den Punkt (zit. in Fricke 1995: 48). Der Gesamtklang lässt nicht mehr an ProberaumMitschnitte denken, wenngleich jeder Titel live eingespielt wurde. Vielmehr wirkt alles darauf angelegt, größtmögliche Energie und Härte, eine erhabene, fast körperlich verletzende Klanggewalt aufzubauen: Im Amphetaminkonsum beschreibenden Titelsong sind Gitarre und der das Klangbild dominierende Bass so sehr übersteuert, wie damals nur möglich, sodass die Gitarre schon mehr ein Rauschen auf dem linken Kanal fabriziert als ein nachvollziehbares harmonisches Spektrum. Das Schlagzeug ist auf dem rechten Kanal gerade eben durch das auf zweiter und vierter Zählzeit geschlagene Becken zu identifizieren. Der Tiefenbereich ist von Gitarre, Bass und Klavier so sehr ausgefüllt, dass man die zusätzliche Verwendung tiefer Trommeln nur vermuten kann. Der Aufnahme wurden offensichtlich keine Overdubs hinzugefügt, da man keinen Anlass sah, typische >Fehler < einer solchen Produktion wie unterschiedliche Lautstärken des Gesangsvortrages durch unbeabsichtigt variierenden Abstand zum Mikrofon nachträglich zu korrigieren. Ohnehin ist der Gesang nicht so in den Frequenzbereich eingepasst, wie man das von professionellen, >glatten< Produktionen gewohnt ist - er wirkt isoliert, eher wie ein Fremdkörper.

All dies gilt - nochmals gesteigert - für das Schlussstück »Sister Ray«, die siebzehneinhalbminütige Improvisation über ein zweitaktiges Riff auf Tonika und Subdominante. Auch hier hatte die Band vor der Aufnahme entschieden, nur einen einzigen Take zu spielen, der ohne Korrekturen gültig sein sollte. Die zwei Gitarren von Morrison und Reed werden über maximal verzerrende Verstärker gespielt, doch den Kampf um die höchste Lautstärke verlieren sie gegen die Orgel John Cales, dessen Spiel weniger durch das verwendete melodische Material als durch die eingesetzten Sounds auffällt. Wie alle anderen Instrumente haben die verschiedenen Orgelklänge sehr hohe Geräuschanteile. Manchmal klingt Cales Orgel hupen- oder sirenenartig, dann wie ein Kratzen oder ein Schlagen gegen Metallplatten. Einige der scharfen, unangenehmen Klänge erinnern an den etliche Jahre später aufkommenden Industrial-Stil, bei dem dann tatsächlich Geräusche von Motorsägen und Schrottplatzfundstücken eingesetzt werden. Wer sich auf die volle Länge dieser Aufnahme einlässt, kann einen brutalen Klangrausch 
erleben, der die auf vergleichbare Wirkungen abzielenden Versuche psychedelischer Musik harmlos erscheinen lässt. ${ }^{3}$

Die Motive für die Klanggewalt der beschriebenen frühen Velvet Underground-Stücke waren vor allem künstlerisch-kritischer Natur: Der Sound ist Mittel des Ausdrucks, denn vor allem durch das Sounddesign der einzelnen Instrumente und die Produktionsweise gewinnt die Musik einen wichtigen Teil ihrer expressiven Qualität. Die extremen Emotionen, von denen Reeds Texte meist handeln, werden durch die radikale Klanggestaltung überzeugend vermittelt, ja spürbar. Konzeptionelles Ziel war darüber hinaus, eine irritierende, provozierende, möglicherweise auch subversive Wirkung der Musik, die ihrem gewohnten Gebrauch zur Unterhaltung entgegenstand: »Ich hatte nicht die Absicht, unsere Musik so zu gestalten, dass sie dem Publikum angenehm war. Wir wollten die Leute wirklich verärgern« so Cale (zit. in Palmer 1997: 198), der damit an ein Motto Warhols anknüpft: »Always leave the audience wanting less « (zit. in Fricke 1995: 19). ${ }^{4}$

Somit nutzten die Gruppe und ihre Hörer die Musik ganz bewusst zur sozialen Distinktion. Ihr Sound demonstrierte im Zusammenspiel mit den Texten sowie den Minimalismen in Rhythmus, Harmonik und Melodik ihre grundlegend ablehnende Haltung zur bürgerlich akzeptierten Kultur einerseits und zur Ideologie von >Love and Peace< sowie der damit verbundenen positiv gestimmten Musik andererseits. Stattdessen präsentierte man sich einer ganz anderen Szene zugehörig, der randständigen intellektuell geprägten Avantgarde.

Ansätze zu stark verzerrten oder feedbackdurchsetzten Sounds gab es unterdessen auch in England. Inwiefern »You Really Got Me« und »All Day And All Of The Night « von den Kinks (beide 1964), »I Wanna Be Your Man« und "Satisfaction« (Rolling Stones, März 1964 und Mai 1965) oder »Anyway Anyhow Anywhere von The Who (Mai 1965) John Cale während seiner Englandreise im Sommer 1965 erst zum radikalen Einsatz solcher Stilmittel inspirierten oder ob sie lediglich Ansporn waren, einen bereits eingeschlagenen Weg weiterzugehen, ist unklar. ${ }^{5}$

3 Noch extremer experimentierte Reed 1975 auf Metal Machine Music mit auf vier Schallplattenseiten ausgedehntem Krach.

4 In dieselbe Richtung zielte ein anderer Ratschlag Warhols: » Lasst es rau klingen. Schleift nicht alle Kanten ab, damit es niemanden mehr stört. ‘ Andy wollte die Leute verstören und aufrütteln - genau wie wir« (Lou Reed, zit. n. Palmer 1997: 198).

5 »The most important things I brought back to New York from this visits were records by the Small Faces and the Who, where noise started showing up. The guitar solo in -Whatcha Gonna Do About It - [der ersten, im August 1965 erschienenen Single der Small Faces] is all crackle and feedback. I said, ,Shit, Lou, we 
Bekannt ist dagegen, dass das Image der frühen Rolling Stones als >dreckiges < und >raues • Pendant der >sauberen< und >netten< Beatles vor allem auf dem Inszenierungsgeschick ihres Managers Andrew Loog Oldham beruhte. Auf der musikalischen Seite ist eine solche Kontrastierung zwar ebenfalls viel zu pauschal (man bedenke das gekrächzte »Twist And Shout « (1963) bei den Beatles oder »Tell Me« (1964) und »Lady Jane« (1965) bei den Stones), doch stützen die ersten Plattenveröffentlichungen eine solche Charakterisierung zumindest hinsichtlich des Sounds. Während die Beatles mit George Martin einen sehr erfahrenen Produzenten an ihrer Seite hatten, trug Stones-Manager Oldham, der (wie Warhol) keinerlei musikalische oder musiktechnische Ausbildung hatte und dem bei den Aufnahmen zur ersten Single »Come On« (1963) selbst grundlegende Studiokenntnisse fehlten, bis zum fünften Album die Verantwortung des Produzenten. ${ }^{6}$ Flexible Klanggestaltungsmöglichkeiten gab es bei den frühen Rolling Stones-Aufnahmen jedoch ohnehin kaum: "We did them on a two-track Revox in a room insulated with egg cartons at Regent Sound. Under those primitive conditions it was easy to make that kind of sound, but hard to a make a much better one « (Keith Richards, zit. in Dalton/Farren 1994: 110f.). Im direkten Vergleich mit der Beatles-Fassung des Lennon/McCartney-Songs »| Wanna Be Your Man« (beide 1963) ist der weitaus härtere, erdige Klang der Rolling Stones-Version mit dem fies-schrillen, an Schmirgelpapier erinnernden Sound der stark verzerrten Sologitarre jedoch nur als bewusst gestaltet zu interpretieren.

Die Stones hatten schon durch ihre stärkere Orientierung an den schwarzen Rhythm'n'Blues-Vorbildern andere Ambitionen und Klangideale als die Beatles, und so bleiben auch spätere Aufnahmen - etwa »Have You Seen Your Mother, Baby, Standing In The Shadow?« (1966) - hinsichtlich der Differenzierbarkeit der einzelnen Instrumente oder des Vermeidens von Geräuschanteilen weit hinter dem nicht zuletzt von den Beatles etablierten Standard zurück. Wohlklang anstrebenden Harmoniegesang oder den Einsatz von Streichquartetten findet man bei den Rolling Stones zu dieser Zeit nicht.

gotta get a deal. They're catching up to us! «, so John Cale (zit. in Fricke 1995: 15). Auf den im Juli 1965 aufgenommenen Demobändern sind noch keine verzerrten Gitarren zu hören, stattdessen aber schrille Instrumentalpassagen von Mundharmonika und Viola.

6 »When engineer Roger Savage turned to him to discuss mixing the two-track recording, sproducer Oldham didn't know what he meant, and promptly instructed the engineer to perform the necessary inconvenience himself (Hector 1995: 1). 
Nachdem 1967 mit dem in Eigenregie produzierten Their Satanic Majesties Request die Antwort der Rolling Stones auf die hochambitionierten Projekte der Beatles und der Beach Boys gemeinhin als missglückt angesehen wurde - es fehlte offensichtlich das Korrektiv eines Produzenten folgten die Rolling Stones 1968 mit dem Album Beggars Banquet und der vorangehenden Single »Jumping Jack Flash« einer Gegenbewegung zu den immer aufwändigeren Studioproduktionen. ${ }^{7}$ Mit hochkulturellem Anspruch, den weite Teile der Pop- und Rockmusik 1966/67 mit dem Einsatz großer Orchester und anderen Kunstmusik-Bezügen erkennen ließen, wollten die Rolling Stones sich nicht identifizieren. Zurück zu den Wurzeln sollte es auf Beggars Banquet gehen, stilistisch ebenso wie bei der Soundproduktion. Besonders deutlich wird dieses Anliegen bei »Street Fighting Man«, für dessen Aufnahme man auf Homerecording-Techniken zurückgriff:

»Zu Beginn einer Session holte Keith seinen neuen Philips-Kassettenrecorder heraus und spielte uns das Demo eines Songs vor, den er zu Hause aufgenommen hatte. Wir versuchten daran zu arbeiten, bekamen aber nicht denselben Sound oder das Feeling wie auf dem Demo hin. Dann holte Charlie einen kleinen antiken Kasten heraus, den er gerade in der Stadt gekauft hatte. Darin befanden sich eine kleine Trommel und ein kleines Becken [bei Hector 1995: 63 als »toy drum kit« beschrieben]. Als er darauf auf dem Boden zu spielen begann, setzte sich Keith mit einer akustischen Gitarre neben ihn, und sie spielten die Nummer gemeinsam. Es klang toll. So bauten sie Keiths Kassettenrecorder auf und versuchten den Song mit dessen einfachem Mikrophon einzufangen. Verglichen mit der Raffinesse der damals bereits üblichen

7 Als erster schlug Bob Dylan, der das Sgt. Pepper-Album für überproduziert hielt, diesen Weg zurück zur Ursprünglichkeit und Einfachheit ein, wenngleich Dylans Alben auch zuvor nicht mit den komplexen Studioarbeiten der Beatles vergleichbar waren. Die im Herbst 1967 in nur neun Stunden aufgenommene LP John Wesley Harding bemüht sich mit starken Country-Anleihen um ein natürliches Klangbild und ist mit Gitarre, Bass und Schlagzeug sehr sparsam instrumentiert: Drummer Kenny Buttrey beschreibt die Aufnahmen: "We went in and knocked 'em out like demos. It seemed to be the rougher the better. He would hear a mistake and laugh a little bit to himself as if [to say], Great, man, that's just great. Just what I'm looking for « (zit. in Sounes 2002: 271, Anm. u. Herv. von Sounes). Kurz nach der Veröffentlichung dieses Albums im Februar 1968 traten auch die Beatles mit dem vergleichsweise reduzierten, an die eigenen R'n'B-Wurzeln anknüpfenden »Lady Madonna« an die Öffentlichkeit. Mit der Arbeit am wiederum Country-beeinflussten Beggars Banquet begannen die Rolling Stones dann im März 1968. Im Mai kamen die Byrds mit einer Country-Rock Single auf den Markt (Dylans »You Ain't Going Nowhere «), im August erschien mit Music From Big Pink das Debüt von Dylans früherer Begleitband The Band, auf dem die Tendenz zurück zum Schnörkellosen, Ursprünglichen prototypisch hervortritt. Im Januar 1969 scheiterten dann die Beatles mit dem programmatisch »Get Back« betitelten Versuch, sich ihrer (Live-)Wurzeln zu besinnen. 
Tontechniken war das eine primitive, >handgestrickte Methode, aber es funktionierte. Der rohe Sound gefiel uns allen sehr« (Wyman/Coleman 1992: 560).

»Parachute Woman« wurde ebenso produziert (vgl. St. Michael 1994: 29) und das, obwohl der Gruppe mit Jimmy Miller für dieses Album erstmals ein namhafter Produzent zur Seite stand, der für eine deutlich bessere Klangqualität als auf allen vorangehenden LPs sorgen sollte. Die Tendenz weg vom Aufwändigen, Komplexen und Kunstvoll-Künstlichen ist übrigens auch bei der Verpackungsgestaltung zu beobachten. Nach den sehr kostspieligen Coverproduktionen von Sgt. Pepper (Peter Blake) und Satanic Majesties (Michael Cooper) wollten die Rolling Stones eine dreckige, bekritzelte Toilettenwand auf dem Cover abbilden. Nach Einschreiten der Plattenfirma erschien Beggars Banquet allerdings einen Monat nach dem so genannten White Album der Beatles ebenfalls in einer schlichten weißen Hülle. Die Korrespondenz ist deutlich: Wer eine Schallplatte mit dem Foto eines vollgeschmierten Klos kauft, darf keinen hochpolierten, sterilen Sound erwarten. Ähnlich hatten bereits im Januar Velvet Underground den Minimalismus von White Light / White Heat mit dünnen weißen Lettern auf schwarzem Grund bildlich umgesetzt.

Die Bezüge zwischen Velvet Underground und den Rolling Stones beschränken sich aber nicht auf derlei Äußerlichkeiten: Mick Jagger sieht die erste Velvet Underground-LP als wichtigen Einfluss für Beggars Banquet:

"Lou Reed started everything about that style of music, the whole sound and the way you play it. I mean, even we've been influenced by the Velvet Underground. [...] I'll tell you exactly what we pinched from him too. Y'know `Stray Cat Blues«? The whole sound and the way it's paced, we pinched from the very first Velvet Underground album. Y'know the sound on >Heroin<. Honest to God, we did!« (Mick Jagger zit. in Dalton/Farren 1994: 108).

Die Parallelen von »Stray Cat Blues« und »Heroin« sind zwar nicht gerade offensichtlich, bei mehrfachem Hören aber doch überzeugend nachzuvollziehen, wenngleich die Stones nicht die Radikalität der Velvet Underground anstreben: Wie »Heroin« beginnt der Stones-Song mit der langanhaltenden Repetition eines einzelnen Gitarrentones. Mit dem ersten Refrain setzt eine Lead-Gitarre mit sehr schrillem, metallischen Klang ein, einem Sound, wie er auch für das Gitarrensolo in »Sympathy For The Devil« verwendet wird. Die Gitarren sind stark verzerrt, das Schlagzeug ist leicht übersteuert aufgenommen. Das letzte Drittel des viereinhalbminütigen Stücks besteht aus einer Stones-untypischen, sich steigernden klangorientierten Improvisation über einen Akkord, die mit ihrem monotonen Minimalismus und der schrillen Lautstärke durchaus an Velvet Underground-Aufnahmen wie »Sister Ray« er- 
innert. So gehört, erscheint Beggars Banquet mit seinen Country- und Delta Blues-Anleihen tatsächlich als »die widernatürliche Verschmelzung von Robert Johnson und Velvet Underground« (Bruckmaier 1999: 37).

Zentrales Album in diesem Sound-Kontext ist aber die 1972 veröffentlichte Doppel-LP Exile On Main St., auf die man, so das Rolling StoneMagazin, heute »jede Platte, die ungeschliffen attackiert und auf jegliche Politur verzichtet«, zurückführen kann (Anonym 1997: 5). Über die Entstehungsbedingungen und den damit verbundenen Sound wurde in blumigen Worten viel geschrieben (vgl. Wild 1997: 8; Bruckmaier 1999: 37). Demnach wurde Exile von Sommer bis Herbst 1971 unter chaotischen Zuständen größtenteils im Keller von Richards' südfranzösischer Villa mit Hilfe eines mobilen Studio-Trucks eingespielt. Jagger war nur unregelmäßig zugegen, Richards befand sich in tiefer Heroin-Abhängigkeit und die extrem hohen Temperaturen machten die meist erst spät abends begonnene Arbeit im modrigen Keller zur Qual. Stilistisch orientierte man sich zum wiederholten Male, diesmal aber mit großer Souveränität, an den verschiedenen Erscheinungsformen (zumeist afro-)amerikanischer Musik: Blues, Rhythm'n'Blues, Soul und Country. In einer treffenden Analyse deutet Adam Olschewski (2002) diese Aufnahmen im selbstgewählten, dekadenten Luxus-Exil auf der Flucht vor den englischen Steuerbehörden als Ziel einer langen Suche nach der Outlaw-Authentizität und der Ursprünglichkeit, die die Stones an ihren schwarzen Delta Blues-Vorbildern so bewunderten: Sie hatten sich ein »Phantasiedelta« geschaffen, voller >schlammiger < Klänge und »angestrebter Verkommenheit«. Die bildreiche Illustration seines Höreindrucks stützt Olschewski vor allem auf den Sound:

»Zwei LP-Seiten lang: ein ungelenker, mal eben so dahingeschmierter Vortrag, durch eine Pappwand aufgezeichnet. Hätte man die Entstehungsgeschichte der beiden Platten nicht gekannt, könnte man auf sie mit Mitteln der Deduktion, mit Hilfe des Hörvermögens gepaart mit einem bisschen Lebenserfahrung schließen. War keiner der Anwesenden bei der Sache? Vielleicht. Aber sie waren, das macht den Unterschied, gemeinsam und gleichzeitig nicht bei der Sache. Man hört also die Hitze, den Schweiß tatsächlich; [...] Man sieht sogar die Feuchtigkeit die Kellerwände entlangrinnen. Mitunter schlägt man die Hände über dem Kopf zusammen wegen des Sounds, der dem Dämon, falls es den gibt, des Chaos' geweiht und dumpf und dennoch erregt fiebrig sich über jedes der 18 Lieder stülpt. Alles taumelt, man verliert rasch die Übersicht: Ist das ein Akkord der passt oder nicht, wirkt das nicht etwas übersteuert... Ein Kollaps droht und doch kollabiert nichts. Ein Klumpen Deltaschlamm, ein Jam, der nicht enden, nicht gerinnen will: man ahnt die Instrumente mehr, als dass man sie sicher identifizieren kann. Jagger, ob- 
wohl manchmal kaum zu verstehen, ist wahrscheinlich vorhanden; ein Piano, ja, müsste wohl auch dabei sein. Ebenfalls eine Gitarre, die ein paar Riffs einstreut, die das Lied dürftig zusammenhalten und arg wankend in Häfen mit Melodienamen tragen, das muss Richards sein. Hat man Glück, dann erwischt man gelegentlich das Schlagzeug von Watts [...]. Man ist im Delta [...]. Schlingpflanzen, Sümpfe, ein Alligator da, nein, dort - alles so wie man es aus dem Naturfilm kennt [...]. Man bietet uns hier die Illusion vom Einblick in den Zustand des Ich wie es das Leben [...] gestaltet, wenn man es willenlos an sich lässt; roh und unbehauen, voll Widerspruch. Mal schön im von Drogenmissbrauch gelenkten Riff, mal gedämpft, wie verschämt fast [...] mal entspannt und düster und erstickend in der Wattigkeit des Sounds. [...] Hermetiker des Loslassens waren hier am Werk« (Olschewski 2002).

Nüchterner betrachtet, muss man feststellen, dass die Rolling Stones auch sehr konzentriert an diesem Album gearbeitet haben. Zahlreiche Overdubs und langwierige Nachbereitungen in Los Angeles widersprechen der romantisierenden Illusion, die Band habe das Album gemeinsam >live aufgenommen. Zudem stammen einige Songs von früheren Aufnahmesessions in London. Richtig ist aber, das bei der Produktion wenig Wert auf Differenzierbarkeit der Instrumente gelegt und eine dicht verwobene, vom natürlichen Raum der Aufnahme geprägte 'wall of sound angestrebt wurde, was zeitgenössische Kritiker auch ohne Kenntnis des legendenumwobenen Entstehungsprozesses in überraschend ähnlichen Bildern beschrieben haben. ${ }^{8}$

Olschewski (2002) hält diese »entschlossen vermurkste Produktion« der Rolling Stones für den »Gipfel ihrer Mühen wie des Rock'n'Roll«, Bruckmaier (1999: 37) spricht vom »besten Album der Rockgeschichte«, und das, obwohl der Sound einhellig als »fiebrig «, »dumpf « und »wattig « beschrieben wird. Produzent Miller soll alles andere als zufrieden gewesen sein, er hätte »dem sumpfigen Sound [...] gerne zu mehr Kontur verholfen« (Anonym 1997). Warum aber wird ein Album, dessen Produktion so den gängigen Ansprüchen widerspricht, gerade wegen dieses Sounds so sehr geschätzt? Was ist der Reiz am miesen Klang?

Nun, das undifferenzierte Klangbild wird gelesen, als würde hier ein Lebensgefühl unmittelbar eins zu eins von der Band auf das Band übertra-

8 »Exile On Main Street is the Rolling Stones at their most dense and impenetrable. In the tradition of Phil Spector, they've constructed a wash of sound in which to frame their songs, yet where Spector always aimed to create an impression of space and airiness, the Stones group everything together in one solid mass, providing a tangled jungle through which you have to move toward the meat of the material. Only occasionally does an instrument or voice break through the surface, and even then it seems subordinate to the ongoing mix « (Kaye 1972: 40f.). Besonders konsequent setzten sie diese Soundabsicht bei »All Down The Line« um, das wie die Spector-Aufnahmen mono abgemischt wurde. 
gen. Ein wildes, hedonistisches, dabei aber schöpferisches Leben, von dem möglicherweise viele insgeheim träumen, das sich jedoch niemand in seiner realen Lebenssituation erlauben kann, an dem man aber zumindest beim Hören dieser Doppel-LP (ähnlich wie im Roman oder Film) teilhaben kann. Ein unvollkommener, spontan oder willkürlich erscheinender Sound wird als höchst authentisch, ausdrucksstark, ungekünstelt, ehrlich gedeutet: hier geht es, so die weit verbreitete romantisierende Rezeption, nicht um Technik, nicht um ein bewusst und rational konzipiertes Produkt, das hohe Verkaufserlöse erzielen soll. Es geht um das Leben, das man während der Aufnahme nicht dem Arbeitsprozess unterwirft, sondern ungebrochen in Musik abbildet, um Ursprünglichkeit, wie man sie bei den afroamerikanischen Vorbildern bewundert, und um Gemeinsamkeit. Wer so lebt, wie man sich Rockstars vorstellt, der kann keine ssauberen< Platten produzieren, denn dazu bräuchte es Disziplin. Stattdessen prägen Lässigkeit und eine »don'tgive-a-shit-Aura (Anonym 1997) das Klangbild - eine Grundhaltung, die man wenige Zeit später auch im Punk nicht lange suchen muss.

Die Punks erklärten all das, was die US-amerikanischen Garagen-Bands, die ersten Velvet Underground-Alben und die >dreckigen< Aufnahmen der Rolling Stones kennzeichnet, zum obersten ästhetischen Prinzip: Punk stand für Schmutz, Simplizität, Unvollkommenheit, Leidenschaft statt Virtuosität, Außenseitertum und Selbermachen aus Überzeugung. Aber während die Punks Rock wieder zur realistischen Ausdrucksform einer Jugendkultur machen wollten, entwickelten sich die Stones zu glamourösen Jet-SetSuperstars und damit zu abschreckenden Beispielen für die Punk-Bewegung: »No Elvis, Beatles or Rolling Stones in 1977 «, so The Clash auf der B-Seite ihrer ersten Single. Den musikalischen Errungenschaften der Stones bleiben die Punks dennoch in gewissem Maße verpflichtet, allen voran die New York Dolls. Zudem erinnerten Malcolm McLarens Imagekonstruktionen für diese Band und für die Sex Pistols an das von Oldham entworfene öffentliche Bild der frühen Stones. Aber auch der unvollkommene Sound der Stones wurde häufig kopiert: »Many of the English punk records sound like our early records, and that sound is very hard to achieve nowadays. But it seems to be the sound many of them are aiming for «, so sieht es Keith Richards (zit. in Dalton/Farren 1994: 110) trotz seiner wenig Objektivität versprechenden Position wohl zu Recht.

Einfacher ließ es sich indes an die kommerziell erfolglosen Velvet Underground anknüpfen, deren Außenseiter-Image viel eher mit der Punkhaltung zu vereinbaren war: »The Velvets hated everything. The whole idea was to take a stab at everything " (Ronnie Cutrone, zit. in Colegrave/Sul- 
livan 2001: 33). Als Bindeglieder können die Detroiter Stooges und MC5 gesehen werden, die bereits 1969 wesentliche musikalische Merkmale des Punk aufwiesen. Iggy Pop, Sänger der Stooges, erinnert sich an den Beginn seiner Velvet Underground-Begeisterung und die Wirkung, die Sound und Produktionsweise ihres Debüts auf ihn hatten:

"I just hated the sound. You know, >How could anybody make a record that sounds like such a piece of shit? This is disgusting! [...] This just sounds like trash! « Then, about six months later, it hit me. >Oh my God! Wow! This is just a great fucking record!^ That record became very key for me, not just for what, and for how great it was, but also because I heard other people who could make good music without being good at music. It gave me hope (Iggy Pop, zit. in Colegrave/Sullivan 2001: 41). ${ }^{9}$

Für die selbstbetitelte erste LP konnten die Stooges 1969 John Cale als Produzenten gewinnen, der nach der Veröffentlichung von White Light / White Heat Velvet Underground verlassen hatte. Er sollte für das Velvet-erprobte, erregt-offensive Klangbild sorgen, das für den Großteil späterer Punkaufnahmen prototypisch wurde: laute, stark verzerrte Gitarren sowie schlichte, naturbelassene Drum-, Percussion- und Stimmklänge ohne große Differenzierbarkeit oder Brillanz, ohne Schmuck und Schnörkel. Von Cale selbst stammen die einzigen Besonderheiten in den Arrangements: auf »I Wanna Be Your Dog « wiederholt er minutenlang einen einzigen Pianoklang, »We Will Fall« wird nach bewährtem Rezept von einem nicht enden wollenden Violaton untermalt.

Des Weiteren sorgte Cale als Produzent für den Sound des 1972 aufgenommenen, aber erst 1976 veröffentlichten Debütalbums von Jonathan Richman and the Modern Lovers, das gelegentlich als eines der ersten Punkalben angesehen wird (vgl. z.B. Strong 1998: 692; Colegrave/Sullivan 2001: 75), und des ebenfalls für die Punkgeschichte bedeutsamen Erstlingswerks Horses von Patti Smith (»a blueprint for a generation of both American and British punk/new wave artists«, Strong 1998: 763). ${ }^{10}$

9 Beide Gruppen hatten Konzerte der Velvet Underground besucht. Entdeckt und gemanagt wurden die Stooges (später auch die Ramones) von Danny Fields, einem Dauergast in Warhols Factory und großem Velvet Underground-Fan.

10 Lenny Kaye, Gitarrist der Patti Smith Group und zuvor Rezensent für den Rolling Stone (vgl. Kaye 1972), war im Übrigen für die in diesen Jahren einsetzende Rückbesinnung auf die Ästhetik des Garagen-Rocks hauptverantwortlich. Selbst Veteran der Garagen-Szene, hatte er diese zwischenzeitlich vollständig vergessene Musik mit der 1972 von ihm kompilierten Doppel-LP Nuggets wieder ins Bewusstsein gebracht. Aus Nuggets wurde eine zwölf Alben umfassende Reihe, welcher der sich kurz darauf ausbreitende Punk wichtige Anstöße verdankt. Mit »Punk « bezeichnete Kaye den Garagen-Rock übrigens schon im Begleittext der ersten Ausgabe. 
Noch stärker auf den nackten Kern reduziert erschien der Garagen/ Velvet Underground/Stooges-Sound bei den New Yorker Ramones: ein verzerrtes, mitten-orientiertes und stark komprimiertes Gitarrenrauschen auf dem rechten Kanal, davon abgetrennt ein ebenso lauter, trockener Bass links, in der Mitte klangtechnisch anscheinend unbearbeitete Drums und klarer, in den Vordergrund gemischter Gesang, das Ganze matt und ohne natürlichen Raumklang aufgenommen: auf dieses einfache Rezept beschränkte sich jeder Song des 1976er Debüts.

Auch in England wurde dieser rohe, laute, dreckige, ungekünstelte Sound kopiert, zumal er hier auf eine wirtschaftlich benachteiligte Jugend traf, deren Lebenssituation er offenbar treffend widerspiegelte. Hier schmückte man sich mit dem, was die Überflussgesellschaft für Müll hielt. Müll sollte auch den Sound prägen, er sollte alle, die nicht dazu gehörten, ebenso abstoßen und ausschließen wie das Äußere der Musiker und Fans.

Nicht viel elaborierter als Proberaummitschnitte klangen die ersten britischen Punk-Singles von The Damned (»New Rose«, Nov. 1976) und den Buzzcocks (»Breakdown«, Jan. 1977) aber auch deshalb, weil man auf professionelles Equipment verzichten musste und wollte. Mit minimalem Etat und meist auch geringen Fähigkeiten bei Musikern und Technikern wurden die Aufnahmen in kürzester Zeit als praktizierte Kritik an standardisierten Einheitsprodukten, die man sich ohnehin nicht leisten konnte, im Do-ityourself-Verfahren mitgeschnitten und vertrieben. Das ergab den einzig passenden, nämlich rauen und aggressiven Ausdruck, den man suchte und zu dem man wirtschaftlich gezwungen war.

Etwas anders verhält es sich mit den Sex Pistols, deren Coverversionen von »No Fun« (1977) und »Roadrunner « (1979) wiederum auf die Stooges und J. Richman verweisen: durch die hohen Summen, die sie als Entschädigung für die Vertragsauflösungen mit den Majors $E M I$ und $A \& M$ gewonnen hatten, stand genug Geld für längere Studiozeit zur Verfügung. Anders als bei The Clash, deren Debüt in nur einer Woche aufgenommen wurde, soll die Produktion von Never Mind The Bollocks, Here's The Sex Pistols (1977) fast ein Jahr gedauert haben (Cunningham 1998: 280). Die Band hatte sich zudem einen namhaften Produzenten, nämlich Chris Thomas, ausgesucht, der bereits für die Beatles, Procul Harum und Roxy Music gearbeitet hatte. Das Album klingt somit durchaus professionell produziert, dabei aber nicht glatt und angepasst. Auffällig ist das dichte, sehr kompakte Klangbild, das durch ausgiebigen Einsatz von Kompressoren und Limitern besonders bei den Gitarren und dem Schlagzeug erreicht wird. Die Gitarren haben eine starke, dabei aber natürliche, warme und obertonreiche RöhrenverstärkerVerzerrung, bei der vor allem die Mittenfrequenzen betont werden (vgl. 
"Seventeen «). Auf diese Weise nimmt die Gitarre kein breites Klangspektrum ein, so dass auch der Bass gut hörbar ist und alle Instrumente recht deutlich voneinander abgehoben sind. Verstärkt wird dieses Hörbild durch die Stereo-Aufteilung, die insbesondere zur räumlichen Differenzierung der Becken genutzt wird. Auch um das dichte Klangbild bei den Refrains zu öffnen, werden Stereo-Effekte genutzt, so bei »Bodies« oder »No Feelings«, wo im Refrain zwei zusätzliche Gitarrenspuren mit starker Stereo-Trennung eingesetzt werden. Die gleiche Wirkung haben die Background-Vocals, die durch starken Raumhalleinsatz sehr entfernt wirken. Der charakteristische Gesang Jonny Rottens steht klar im Vordergrund. Hier wurde offensichtlich nichts dem Zufall überlassen, um eine immer wieder hörbare, technisch den Radiostandards entsprechende Qualität zu erzielen, ohne klanglich die Punknormen allzu sehr zu verletzen. Zudem verhindern shingerotzter A AntiGesang und skandalträchtige Texte Authentizitätszweifel an den präzise und diszipliniert eingespielten, handwerklich guten Aufnahmen.

Die musikalischen Beschränkungen des Punk wurden schnell inflationär und somit ausdruckslos und überkommen. Da Minimalismus und unprofessionelle Produktion nun kein Aufsehen mehr erregten, taugten sie nicht einmal mehr zur individuellen Abgrenzung. Die an den Punk anknüpfende New Wave verfolgte daher zwar eine Punk-ähnliche Ideologie, veränderte aber den Sound grundlegend. Nun galt ein kaltes, steriles oder technoides Klangdesign mit seinen Assoziationen ans Industrielle, Unpersönliche und damit Entfremdete als geeignet, um eine gesellschaftskritische Haltung akustisch zu unterfüttern.

Besonders radikal war in diesem Kontext das Album Psychocandy von The Jesus and Mary Chain (1985), auf dem mit traditionellem Rock-Instrumentarium ein noch stärkerer Klang-Terror produziert wird als bei "Sister Ray«. Der Reiz besteht dabei in der Kontrastierung von ssüßen Melodien und extremem Sound, der Journalisten zu Beschreibungen wie »Beach Boys mit einer Kreissäge im Hintern " oder "Staubsauger außer Kontrolle veranlasst hat (zit. in Graves et al. 1998: 461). Mehrere Komponenten tragen dazu bei: Auffälligstes Merkmal sind die lauten Gitarren, deren Klang mit Distortion-Effekten höchstmöglich verfremdet wird. Dabei wird keine warme Röhrenübersteuerung eingesetzt, sondern eine kalte, blecherne, den oberen Frequenzbereich stark betonende Verzerrung nahe am weißen Rauschen. Die Assoziationen sind vielfältig: man könnte an industrielle Metallschneidearbeiten, an Testbildrauschen oder einen Sandstrahl denken. Selbst Akustikgitarren werden hässlich verzerrt ("Some Candy Talking "). Dazu gibt es reichlich Rückkopplungen, teilweise sogar durchgängige Feedback-Teppiche 
(»Never Understand«). Vom Schlagzeug ist häufig nur die Snare-Drum zu hören, von deren Frequenzspektrum meist nur ein sehr schmaler, mittlerer Bereich verstärkt wird und auf die ein extremer, manchmal sogar sekundenlanger Nachhall gelegt wird (»In A Hole«, »Sowing Seeds«). Auch der Gesang ist mit sehr viel künstlichem Hall verfremdet, so dass die Stimme Jim Reids wie aus der Ferne oder in einer großen, leerstehenden Fabrikhalle aufgenommen klingt.

Die Idee hinter diesem Sound ist offensichtlich: Er ist das wirksamste Mittel, um eine melancholische Grundstimmung von Isolation, Entfremdung, Kälte und Unnatürlichkeit überzeugend zum Ausdruck zu bringen. Von etwa 1989 bis 1992 folgten zahlreiche (als >Shoegazer b bezeichnete) Bands, unter ihnen My Bloody Valentine (vgl. den Beitrag von Ruben Jonas Schnell in diesem Buch), Ride, Lush oder die Boo Radleys, diesem in Großbritannien sehr populären Soundkonzept.

Gestützt auf das Do-it-yourself-Prinzip des Punk und das damit etablierte, von den großen Plattenfirmen und ihren Standards unabhängige Vertriebnetz entwickelte sich in den folgenden Jahren ein Stilbereich, für den das ästhetische Prinzip der minimal gehaltenen Produktion und der unprofessionellen Klangqualität so zentral war, dass er sich darüber definierte: die so genannte Lo-Fi-Bewegung. Low Fidelity, also geringe Klangtreue, wiesen die Aufnahmen von amerikanischen Gruppen wie Beat Happening, Pussy Galore, Jandek oder den Neuseeländern Tall Dwarfs (vgl. den Beitrag von Joachim Hentschel in diesem Buch), The Clean und The Chills auf, weil sie mit einfachsten Mitteln auf dem heimischen Vier-Spur-Recorder produziert und zunächst nur auf selbst kopierten Kassetten verbreitet wurden (vgl. Unterberger o.J.[b]). ${ }^{11}$ Trotz der Ablehnung von Professionalität, Vermarktung und Startum, denen einige Bands mit sich ständig ändernden Projektnamen und Labelwechseln sowie dem Verzicht auf Werbung entgegenzuarbeiten versuchten, gewann diese Bewegung 1992/93 (dann schließlich doch auf CD) mit Pavement, Sebadoh, Guided by Voices, Smog sowie den Projekten Will Oldhams an Beliebtheit, Verbreitung und Einfluss. Auch der »prototypische, muffig produzierte Indie-Rock« (Anonym 2003: 66) von Liz Phair, deren Debüt Exile In Guyville (1993) sich konzeptuell an das Exile-Album der Rolling Stones anlehnt, und die frühen Alben von Beck Mellow Gold, Stereopathic Soul Manure (beide 1994), One Foot in the Grave (1995) und Odelay (1996) wurden trotz Lo-Fi-Ästhetik z.T. beachtliche Verkaufserfolge.

11 Auf einer solchen Kassette spielen Pussy Galore übrigens das Exile On Main St.Album Song für Song nach. 
Der Verzicht auf ausgefeilte Studiotechnologie entsprang bei einigen Gruppen sicher der banalen Tatsache, dass sie keinen Major-Plattenvertrag bekommen konnten und den Geldmangel zur Tugend stilisierten. Die Lo-FiHaltung gründete sich aber auch auf eine bewusste Verweigerungshaltung gegenüber der Tonträgerindustrie und ihren Mechanismen, eine Haltung, die auf die beschriebene Tradition aufbaut und sich insbesondere aus dem Punk-Ethos speist. Musik, die nicht den gegenwärtigen Produktionsstandards entspricht, lässt sich schließlich nicht zur Supermarktbeschallung oder für die Fernsehwerbung funktionalisieren. Somit kann Lo-Fi mit seinen Homerecording-Verfahren und den unübersichtlichen Veröffentlichungsstrategien auf Kleinstlabeln als »letzte Bastion gegen die Majors, die sich den alternativen Rock fast vollständig einverleibt hatten« (Buss 1997: 8), und als Reaktion auf die von der Industrie forcierte Durchsetzung der Compact Disc gesehen werden. Zudem versprach man sich von einfach gehaltenen Aufnahmen ohne langwierige Overdubs größere Spontaneität und emotionale Intensität. Solche >nackte<, als >ehrlich` gedeutete Musik sollte den Blick auf das für wesentlich gehaltene Songwriting und den emotionalen Ausdruck lenken, man wollte beweisen, dass die Musik im Gegensatz zu weiten Teilen des hochtechnisierten Mainstream auch ungeschminkt, ohne dicke Schichten Studio-Technik Qualität und Bestand hat. Vorbild für diese Haltung war u.a. Neil Young, der mit live eingespielten, rauen Alben wie Time Fades Away (1973), Tonight's The Night (1975) oder Rust Never Sleeps (1979) des Öfteren die Forderungen der Industrie ignoriert hatte und dafür als höchst authentischer, ausdrucksstarker und eigensinniger Künstler (»godfather of Grunge $\ll$ ) verehrt wurde. ${ }^{12}$

12 Die genannten, schwer konsumierbaren Alben waren eine bewusste Gegenreaktion Youngs auf den großen wirtschaftlichen Erfolg des sglatten< HarvestAlbums (1972): "[»Heart Of Gold«] put me in the middle of the road. Travelling there soon became a bore so I headed for the ditch. A rougher ride but I saw more interesting people there (Linernotes zum Album Decade, 1977). Diese Notizen und viele weitere Coverbeschriftungen auf Neil Young-Veröffentlichungen sind handschriftlich, sicher keine zufällige Korrespondenz zur angestrebten Authentizität der Musik (s. auch die Covergestaltung von Exile On Main St. oder Metallicas Garage Days Re-Revisited, 1987, und Garage Inc., 1998). An Perfektion liegt Young offenbar gar nichts, so dass er viele Songs in einem Take ohne Overdubs aufnimmt (vgl. das in vier Tagen mit Pearl Jam eingespielte Album Mirror Ball, 1995, welches mit der programmatischen Aufforderung »No tuning, nothing! « beginnt). Improvisation spielt dabei eine große Rolle, sieht Young seine Musik doch eher als Prozess, als »something mutable and vital rather than frozen and fixed « (McLeese 1992: 327). So veröffentlicht Young auch auf regulären LPs Live-Mitschnitte, die teilweise klingen, als wären sie mit einem sehr billigen Kassettenrecorder im hinteren Teil der Halle mitgeschnitten worden (vgl. »Baby What You Want Me To Do« auf Broken Arrow, 1996). Als Verfechter der analogen Klangwiedergabe hat Young einige seiner LPs jahrzehn- 
Der Anspruch auf künstlerische Integrität, das Bedürfnis nach Authentizität war also das wichtigste Charakteristikum der Musiker und Hörer der Independent-Bewegung in den 1990er Jahren. Amateurhafte Aufnahmen, mit denen keine großen Gewinne zu erzielen waren, wurden zum Symbol dieser Haltung.

Gerade die Alben von Nirvana, der erfolgreichsten und einflussreichsten Band in diesem Kontext, sind bezüglich der Produktionsweise sehr aufschlussreich: Noch stärker als auf Nevermind The Bollocks... von den Sex Pistols wandelte Nevermind, die erste Major-Veröffentlichung aus dem Jahr 1991, auf dem schmalen Grat zwischen rauer Grunge-Ursprünglichkeit und aufwändig gestaltetem, radiotauglichen Klangdesign. Während die Basisspuren der Songs »Polly« und »Something In The Way« im Lo-Fi-Verfahren mit einer ungestimmten »ultrabilligen, fünfsaitigen Akustikgitarre« mit »schepprige[m] Sound« (Butch Vig, zit. in Di Perna 1997: 71) im Abhörraum des Studios aufgenommen wurden und Cobain für »Territorial Pissings « gegen den Protest des Produzenten die Gitarre ohne Verstärker direkt an das Mischpult anschloss (vgl. Azerrad 1994: 189), fallen vor allem die Singles "Smells Like Teen Spirit« und »In Bloom« durch den druckvoll-bombastischen Drum-Sound, mehrfach übereinandergelegte Gitarrenspuren und gedoppelten Gesang auf. Nirvanas Wildheit und Intensität wurden mit ausgefeilten Studiotricks verstärkt. Das kraftvolle Resultat der Arbeiten von Butch Vig war mit über zehn Millionen verkauften Exemplaren ein großer kommerzieller Erfolg und galt dennoch zugleich als authentischer Ausdruck der Musiker und ihrer ganzen Generation. Die Produktion war ein Kompromiss, aus der Nirvanas Dilemma zwischen Punkideologie und Pop-Appeal spricht: „Nevermind was really raw and punk-sounding for a major-label record. Sure, Butch Vig meticulously labored over each track, but certainly, nothing like this had ever attained such popularity« (Azerrad 1996: 95). Im Nachhinein war Kurt Cobain jedoch überhaupt nicht zufrieden: »Auf eine gewisse Weise fühle ich mich andauernd schuldig. Unsere Musik klingt so glatt, vor allem auf dieser Platte. Um ganz ehrlich zu sein - noch vor ein paar Jahren hätte ich diese Band gehasst« (Cobain, zit. in Di Perna 1997: 46). Später wurde Cobain noch deutlicher: »Wenn ich mir Nevermind anhöre, bekomme ich [...] einen Hass auf die Produktion « (Cobain, zit. in Azerrad 1994: 354, vgl. auch 196f., 330 u. 360).

telang nicht auf $C D$ veröffentlichen lassen. Zu seiner Einstellung gegenüber den »record company men« vgl. »Prisoners« (u.a. auf Year Of The Horse, 1997). Zum Einsatz von Rückkopplungen und Verzerrung vgl. Ragged Glory (1990) und die halbstündige Feedback-Collage Arc (1991). 
Da sie durch den Erfolg eine souveränere Position gegenüber der Plattenindustrie einnehmen konnten, verpflichteten Nirvana für das Nachfolgealbum In Utero (1993) den Underground-Produzenten Steve Albini, der für einen >persönlicheren< und >urwüchsigeren< Sound sorgen sollte. Zu diesem Zweck wurden die Songs innerhalb von nur zwei Wochen größtenteils live aufgenommen und in einer weiteren Woche an einem alten analogen Mischpult gemixt. Inspiriert von den Lo-Fi-Bands Pavement und Sebadoh sowie der eigenen Punk-Vergangenheit verzichtete man weitgehend auf Effektgeräte und Overdubbing, stattdessen sollten Gesang und Schlagzeug von natürlichem Raumklang geprägt sein (vgl. ebd.: 333f.). Im Vergleich zu Nevermind klingen einige Songs, z.B. "Serve the Servants« oder »Milk It « offensichtlich so, wie es die Band zunächst beabsichtigt hatte: "an uncompromising wall of noise « (Strong 1998: 579). Zufrieden war man aber auch diesmal nicht, so dass zumindest die Singles »Heart Shaped Box« und »All Apologies « komplett neu abgemischt und die Lautstärken der Gesangsspuren bei allen Stücken angehoben wurden. Nach Albini sollten die Aufnahmen noch weitaus ungeschliffener klingen, doch kommerziellen Selbstmord wollten Nirvana offenbar nicht begehen. Die Produktionskosten waren mit $130.000 \$$ letztlich ebenso hoch wie bei Nevermind.

Die Konjunktur des >dreckigen Sounds Fragmente des Lo-Fi-Konzeptes bildeten zwar vordergründig die Basis für die populäre MTV Unplugged-Reihe, doch mussten die ursprünglichen Ideale den kommerziellen Interessen des Musikfernsehens weichen. HipHop und Techno, beide stärker als die klassische Rockmusik auf hoch entwickelte Studiotechnik angewiesen, bestimmten für längere Zeit das popmusikalische Geschehen. Möglicherweise als Gegenreaktion auf die großen Erfolge von Stars wie Britney Spears, singenden Schauspielern oder gecasteten Boy- und Girlgroups, deren Musik auf ein immer jüngeres Zielpublikum zugeschnitten wird und deren Sound-Design somit vor allem Gefälligkeit anstrebt, widmen sich Kritiker und Musikmagazine seit dem Sommer 2001 wieder verstärkt der Renaissance einfachen Gitarrenrocks, der nun von denen gespielt wird, die ihre musikalische Prägung von Punk, Grunge und Britpop erhalten haben.

Den Strokes galt die erste Aufmerksamkeit der Medien, The White Stripes, Black Rebel Motorcycle Club, Interpol, The Datsuns, The Hives, The Vines oder The Libertines folgten. Die Korrespondenzen von Sound und Image 'wiederholen s sich dabei ebenso wie die Musik, für die die Kommentatoren zu Recht Velvet Underground, Rolling Stones, Stooges, Ramones, Television, The Jesus and Mary Chain oder Nirvana als wesentliche Vorbilder ausmachen (vgl. Hentschel 2002: 60-65). Auffällig ist, dass diese Bands 
weniger Eindruck in den Hitparaden hinterlassen als bei den stets auf Distinktion vom Mainstream bedachten Musikjournalisten, die hier die Musik ihrer Jugend in neuem Gewand vorfinden:

»Die Strokes sind natürlich die Retter des so genannten Musikjournalisten, denn jetzt scheppert das Schlagzeug wieder, der Bass wummert, und die Gitarren, jawohl, sie mäandern. Oder umgekehrt! Casablancas Gesang aber hat den drawl, man könnte auch sagen: Er singt in ein sehr altes Mikrofon, fast ein Megafon, weshalb er fern und lässig klingt. So klingt die ganze Platte: fern und lässig. [...] Elf Songs, kurz, ruppig, schmutzig, laut, unbedingt, begehrlich, roh, sexy, sinister, unwiderstehlich« (Willander 2001: 92).

Der Sound von Is This It (2001), dem Debüt der Strokes, lehnt sich ganz offensichtlich an Produktionen anderer New Yorker Bands aus den 1970er Jahren an. Herausgestellt werden oft Parallelen zu Television, was bezüglich der ausgefeilten Interaktion von Gitarren und Bass zutrifft. Doch verglichen mit Is This It klingt Marquee Moon (1977) von Television frischer, vielfältiger arrangiert und mit mehr Aufwand produziert. Rein klangtechnisch zitieren The Strokes eher den Ramones-Minimalismus. Erreicht wird dieser altmodische, im Jahr 2001 aber Aufsehen erregende Effekt durch eine muffige, höhenarme Abmischung. Der Frequenzgang der Snare ist sehr eng und mittenbetont (vor allem bei »Soma«), was an Aufnahmen mit alten Mikrofonen erinnert. Gleiches gilt für den Gesang, der auf allen Stücken leicht übersteuert, wie durch ein Megafon klingt. Die Becken, die nicht sehr stark verzerrten Gitarren und der Bass, der wie bei den Ramones im Verhältnis zu den Gitarren recht laut ist, haben einen warmen Klang ohne große Präsenz. Es wird präzise gespielt, das Klangbild ist durch die Stereoverteilung der Instrumente differenziert. Durch Zurufe zwischen den Musikern während der Breaks (»Someday«) oder nicht unterdrücktes Verstärkerbrummen und Feedback (»New York City Cops«) wird ein Live-Eindruck erweckt. Dazu passt, dass die Band in ihren Videos offensichtlich live spielt, statt zum Playback zu mimen. Zu einem solchen Sound, das hat uns die bisherige Betrachtung gelehrt, gehört das passende Image:

»Was die Band auszeichnet, [ist] die ungespielte Rohheit und Glaubwürdigkeit der young offenders, die sich bereits im komplett abgefuckten Booklet der Debüt-Platte niederschlägt: Auf den dort abgebildeten Fahndungsfotos versprühen [die Musiker] den unbehauenen Charme von Schwerverbrechern, die man nach einer durchzechten Nacht zum Fototermin aufs Revier gezerrt hat « (Wigger 2001: 14).

Wem der Sound nicht dreckig genug erscheint, der wird auf die englischen Libertines (Up the Bracket, 2002) verwiesen, die kritisieren, dass Is This It 
mit dem Programm ProTools, also digital produziert worden ist (vgl. Hentschel 2002: 62). Dementsprechend lassen sie sich vom Clash-Gitarristen Mick Jones vorgeblich komplett live und analog aufnehmen. Die Songtitel werden für die Coverrückseite auf einen unscharfen Schnappschuss gekritzelt, während die Vorderseite die ausgeschnittenen Erpresserbrief-Typen der Sex Pistols aufgreift. Die Kritiker sind begeistert ob so viel Purismus und Ursprünglichkeit: »Der Unterschied zu den Strokes besteht darin, dass alles nicht so blitzsauber heruntergespielt wird« (Weiland 2002: 46), »fast jedes Stück eine hingerotzte Attacke« (Brüggemeyer 2002: 97), »diese Band ist wohl die ungewaschenste, die ich je gesehen habe« (Müller 2002: 129). Man sieht: der Kreis schließt sich, Musik, Imagekonstruktionen und die Vorlieben der Hörer > wiederholen< sich.

Vollständigkeit kann in einem solchen Rahmen nicht angestrebt werden, und bei der Auswahl der Beispiele lässt sich die Subjektivität des Betrachters nicht verbergen. Doch worum es im Kern geht, das hätte auch an anderen Beispielen, etwa an Neil Young (s. Anm. 12), Bruce Springsteen (Nebraska), Bob Dylan (The Basement Tapes), Metallica (Garage Inc.; St. Anger), Sonic Youth (Dirty) oder den White Stripes (White Blood Cells; Elephant) gezeigt werden können:

Das Sounddesign ist in der Pop- und Rockmusik zu einem wesentlichen Feld der kreativen Gestaltung wie auch des ästhetischen Erlebnisses geworden; dem Sound verdankt sie einen Großteil ihres kommunikativen Potentials. Dabei kann man tendenziell eine stärker sinnlich-unmittelbare und eine stärker rational-mittelbare Kommunikation unterscheiden: zum einen ist Sound Medium des emotionalen Ausdrucks, er lässt eine spezifische, mit Worten nicht vollständig beschreibbare Atmosphäre entstehen und kann ganze Lebenshaltungen sinnlich vermitteln. Zum anderen ist Sound aber auch Symbol für wichtige außermusikalische Bedeutungen, die innerhalb einer kulturellen Praxis entstehen und erst rational (wenngleich zumeist unbewusst) mit dem nötigen kulturellen Kapital interpretiert werden können. In dieser Funktion wird die Klanggestaltung eingesetzt, um Aussagen über die soziale Position des Musikers zu treffen. So kann man über die Wahl des Klangbildes versuchen, sich als natürlich und ehrlich, also authentisch darzustellen. Man kann mithilfe eines geeigneten Sounds Anspruch auf die begehrte Rolle des Künstlers erheben und ein im weiteren Sinne politisches Statement abgeben, indem man sich z.B. marktwirtschaftlichen Ansprüchen widersetzt oder anpasst. Man kann sich über Sound in eine bestimmte Tradition stellen und sich zugleich von ungewünschten Hörerkreisen distanzieren. Diese Möglichkeiten stehen dabei sowohl dem produzierenden Musiker wie 
auch dem auswählenden und sich dadurch positionierenden Rezipienten offen - ein Kommunikationsweg, der keineswegs eindeutig dechiffrierbar ist: gerade aufgrund der Abstraktheit und schwierigen Verbalisierbarkeit bietet sich Sound (auf Basis der jeweiligen Situiertheit und der bisherigen Praxis) als Projektionsfläche für individuelle Deutungen an.

Die aufgezeigte Bevorzugung eines als dreckig, rau, unvollkommen, kantig, organisch, lebensnah interpretierten Sounds vor seinem als glatt poliert, steril-sauber, geschliffen, unrealistisch, fehlerfrei und angepasst diffamierten Pendant verweist auf ein Bedürfnis nach Natürlichkeit, Echtheit, Menschlichkeit und Individualität. Einer solchen Werthaltung soll hier nicht das Wort geredet werden. Man mag sie je nach Persönlichkeit als einzig richtige oder als konservativ->rockistisch<, romantisierend und altmodisch ansehen. Oder beides.

\section{Literatur}

Anonym (1997). »The Rolling Stones. Exile On Main Street. « In: Rolling Stone (dt.), Nr. 36 (Okt.), S. 5 der Sonderbeilage »Die musikalischen Meilensteine der '70er «. Anonym (2003). "Liz Phair. Whip Smart « [Rezension]. In: Rolling Stone (dt.), Nr. 100 (Feb.), Sonderbeilage »Die 100 besten Alben aus 100 Heften«, o. Seitenzählung.

Azerrad, Michael (1994). Nirvana. Come as you are. St. Andrä-Wördern: Hannibal.

Azerrad, Michael (1996). »Nirvana. Nevermind.«In: Guitar World 16, No. 2 (Feb.), S. 95.

Bangs, Lester (1992). »Protopunk: The Garage Bands.« In: DeCurtis/Henke 1992: 357-361.

Bruckmaier, Karl (1999). Soundcheck. Die 101 wichtigsten Platten der Popgeschichte. München: Beck.

Brüggemeyer, Maik (2002). »The Libertines. Up the Bracket« [Rezension]. In: Rolling Stone (dt.), Nr. 97 (Nov.), S. 97.

Buss, Christian (1997). »Adventures in lo-fi.«In: Rolling Stone (dt.), Nr. 38 (Dez.), S. 8 der Sonderbeilage »Die musikalischen Meilensteine der ' $90 \mathrm{er}$ «.

Colegrave, Stephen / Sullivan, Chris (2001). Punk. London: Cassell \& Co.

Cunningham, Mark (1998). Good Vibrations. A History of Record Production. London: Sanctuary.

Dalton, David / Farren, Mick (1994). Rolling Stones in Their Own Words. London u.a.: Omnibus.

DeCurtis, Anthony / Henke, James (Hg.) (1992). The Rolling Stone Illustrated History of Rock'n'Roll. London: Plexus.

Fricke, David (1992). »The Velvet Underground.«In: DeCurtis/Henke 1992: 348356.

Fricke, David (1995). Begleittext zum Box-Set The Velvet Underground - Peel Slowly and See (Polydor Chronicles).

Graves, Barry et al. (1998). Das neue Rocklexikon. Reinbek: Rowohlt. 
Hector, James (1995). The Complete Guide to the Music of The Rolling Stones. London u.a.: Omnibus.

Hentschel, Joachim (2002). »Rock is back! The Libertines geben dem britischen Punk- und Beat-Erbe den neuen Kickstart.« In: Rolling Stone (dt.), Nr. 98 (Dez.), S. 60-65.

Kaye, Lenny (1972). ">Tumbling Dice< puts the cherry on the first side of >Main Street « [Rezension]. In: Rolling Stone, No. 112 (July 6), S. $40 f$.

McLeese, Don (1992). »Neil Young. «In: DeCurtis/Henke 1992: 324-331.

Müller, Sonja (2002). »The Libertines. Up the Bracket« [Rezension]. In: Intro, Nr. 100 (Nov.), S. 129.

Olschewski Adam (2002). "Seht sie die schlechten Zähne fletschen. Vierzig Jahre Rolling Stones, dreißig Jahre >Exile on Main Street ««In: Frankfurter Rundschau, 26. Oktober, S. 21.

Palmer, Robert (1997). Rock \& Roll. Die Chronik einer Kulturrevolution. St. AndräWördern: Hannibal.

Di Perna, Alan (1997). »Smells Like Teen Spirit. Die >Nevermind --Sessions. «In: Rolling Stone (dt.), Nr. 38 (Dez.), S. 71.

Sounes, Howard (2002). Down the Highway. The Life of Bob Dylan. London: Black Swan.

St. Michael, Mick (1994). Keith Richards in His Own Words. London u.a.: Omnibus.

Strong, Martin C. (1998). The Great Rock Discography. Frankfurt/M.: Zweitausendeins.

Unterberger, Richie (o.J.[a]). »Garage Rock.«In: www.allmusic.com (Zugriff: 13.1. 2003).

Unterberger, Richie (o.J.[b]). »Lo-Fi.«In: www.allmusic.com (Zugriff: 27.1.2003).

Weiland, Thomas (2002). »The Libertines. Up the Bracket« [Rezension]. In: WOM Journal, Nov., S. 46.

Wigger, Jan (2001). »New York City Kings. « In: Rolling Stone (dt.), Nr. 83 (Sept.), S. 13-15.

Wild, David (1997). »Exile On Main Street.«In: Rolling Stone (dt.), Nr. 36 (Okt.), S. 8 der Sonderbeilage »Die musikalischen Meilensteine der '70er «.

Willander, Arne (2001). »The Strokes. Is This It« [Rezension]. In: Rolling Stone (dt.), Nr. 83 (Sept.), S. 92.

Wyman, Bill / Coleman, Ray (1992). Stone alone. Die Insidergeschichte der Rolling Stones. München: Goldmann. 